BNL-HET-01/5

CERN-TH/2001-037

hep-ph/0102117

\title{
Higgs Production and Decay in the MSSM: Status and Perspectives
}

\author{
S. HEINEMEYER ${ }^{1 *}$ AND G. WEIGLEIN ${ }^{2 \dagger}$ \\ ${ }^{1}$ HET, Brookhaven Natl. Lab., Upton, New York 11973, USA \\ ${ }^{2}$ CERN, TH Division, CH-1211 Geneva 23, Switzerland
}

\begin{abstract}
The theoretical predictions in the MSSM for Higgs-boson production at a future $e^{+} e^{-}$Linear Collider and Higgs-boson decay processes are discussed focusing in particular on recent diagrammatic two-loop results in the MSSM Higgs sector. The present status of the theoretical predictions is briefly summarized, and it is emphasized that considerable improvements will be necessary in order to match the high experimental accuracy achievable at a future Linear Collider.
\end{abstract}

*) email: Sven.Heinemeyer@bnl.gov

†) email: Georg.Weiglein@cern.ch 


\title{
Higgs production and decay in the MSSM: status and perspectives
}

\author{
Sven Heinemeyer* and Georg Weiglein ${ }^{\dagger}$ \\ *HET, Brookhaven Natl. Lab., Upton, New York 11973, USA \\ ${ }^{\dagger}$ CERN, TH Division, CH-1211 Geneva 23, Switzerland
}

\begin{abstract}
The theoretical predictions in the MSSM for Higgs-boson production at a future $e^{+} e^{-}$Linear Collider and Higgs-boson decay processes are discussed focusing in particular on recent diagrammatic two-loop results in the MSSM Higgs sector. The present status of the theoretical predictions is briefly summarized, and it is emphasized that considerable improvements will be necessary in order to match the high experimental accuracy achievable at a future Linear Collider.
\end{abstract}

\section{INTRODUCTION}

Exploring the Higgs sector of the electroweak theory with high precision will be one of the main goals of a future $e^{+} e^{-}$Linear Collider (LC). It will allow a highly sensitive test of the investigated theory and will thus provide a way for distinguishing between different models.

A firm prediction of the Minimal Supersymmetric Standard Model (MSSM) is the existence of a light neutral Higgs boson. The Higgs sector of the MSSM consists of two doublets and besides the gauge couplings is described by two parameters, conventionally chosen as the ratio of the two vacuum expectation values, $\tan \beta=v_{2} / v_{1}$, and the mass of the $\mathcal{C} \mathcal{P}$-odd Higgs boson, $M_{\mathrm{A}}$. The masses of the other Higgs bosons and the mixing angle in the neutral Higgs sector are predicted in terms of these parameters, giving rise to the well-known result that the mass of the lightest $\mathcal{C P}$-even Higgs boson, $m_{\mathrm{h}}$, in the MSSM has to be smaller than the Z-boson mass at lowest order. This bound, however, receives large radiative corrections [1], in particular from the Yukawa sector of the theory, which shift the bound to $m_{\mathrm{h}} \lesssim 135 \mathrm{GeV}$ including two-loop corrections [2].

The prospective experimental accuracies at a future Linear Collider in the energy range $500 \mathrm{GeV}-1 \mathrm{TeV}$ will make it necessary to reduce the theoretical uncertainties of the predictions for the Higgs production cross sections and branching ratios below the level of $1 \%$. This goal will be very difficult to achieve, owing to the fact that there exist several different sources for sizable higher-order corrections and for large theoretical uncertainties. 
As mentioned above, large Yukawa corrections affect the predictions for the Higgs-boson masses and the mixing angle $\alpha$. The leading terms at one- and twoloop order are of $\mathcal{O}\left(G_{\mu} m_{\mathrm{t}}^{4} / M_{\mathrm{W}}^{2}\right)$ and $\mathcal{O}\left(G_{\mu} \alpha_{\mathrm{s}} m_{\mathrm{t}}^{4} / M_{\mathrm{W}}^{2}\right)$, respectively. Besides these corrections, which originate from the $\mathrm{t}-\tilde{\mathrm{t}}$ sector of the MSSM, for large values of $\tan \beta$ and the Higgs mixing parameter $\mu$ also large corrections in the $\mathrm{b}-\tilde{b}$ sector are possible, which in some regions of the parameter space can even invalidate a perturbative treatment. Corrections from the scalar quarks of the third generation can also be a source for large loop-induced effects in the Higgs sector connected to complex phases. Concerning theoretical uncertainties related to large loop corrections, in the energy range of a LC genuine vertex and box corrections in general give rise to much bigger effects than at LEP energies, where often the bulk of the weak and QCD corrections originates from universal propagator-type contributions.

In the parameter regions where the widths of the MSSM Higgs bosons are large, an accurate treatment of the Higgs-boson production furthermore requires to take into account off-shell effects, i.e. to study the full $2 \rightarrow 4$ process including nonresonant contributions.

In addition to the above-mentioned effects, a different source of theoretical uncertainties is related to the experimental errors of the input parameters. Owing to the large corrections from the $\mathrm{t}-\tilde{\mathrm{t}}$ sector, in particular an accurate measurement of the top-quark mass is crucial for precise theoretical predictions in the Higgs sector of the MSSM.

\section{THE $\mathcal{C} \mathcal{P}$-EVEN HIGGS-BOSON MASSES AND $\alpha_{\text {eff }}$}

The prediction for the lightest $\mathcal{C P}$-even Higgs-boson mass in the MSSM has recently been improved by the inclusion of non-logarithmic genuine two-loop contributions obtained via an explicit Feynman-diagrammatic (FD) calculation $[2,3]$. These corrections gave rise to a numerically sizable shift compared to the results previously obtained via a renormalization-group-improved one-loop Effective Potential approach (EPA) [4,5]. Recently further sub-leading two-loop electroweak contributions have been obtained [6].

The current theoretical uncertainty in $m_{\mathrm{h}}$ from unknown higher-order contributions can conservatively be estimated as $\Delta m_{\mathrm{h}}^{\text {theo }} \approx \pm 3 \mathrm{GeV}$. This uncertainty is smaller than the one induced from the present experimental error on $m_{\mathrm{t}}$, as a change of $\Delta m_{\mathrm{t}}= \pm 5 \mathrm{GeV}$ leads to a shift in $m_{\mathrm{h}}$ of $\Delta m_{\mathrm{h}}^{\text {theo }} \approx \pm 5 \mathrm{GeV}$ [7]. These uncertainties have to be compared with the prospective accuracies at a future LC of $\Delta m_{\mathrm{h}}^{\exp }=0.05 \mathrm{GeV}$ and $\Delta m_{\mathrm{t}}^{\exp }=0.1-0.2 \mathrm{GeV}$.

The theoretical uncertainties in the Higgs propagator corrections affect the predictions for the Higgs production and decay processes via their effects on $m_{\mathrm{h}}, m_{\mathrm{H}}$, and on the effective mixing angle $\alpha_{\text {eff }}$ in which the bulk of the Higgs propagator corrections to the Higgs couplings can be absorbed.

The two-loop Higgs propagator corrections evaluated in Refs. [2,3] have recently been incorporated into the diagrammatic one-loop result for the QED and QCD 
contributions [8] to the decay processes $h \rightarrow f \bar{f}[9]$ and into the complete diagrammatic one-loop result [10] for the production processes $e^{+} e^{-} \rightarrow h Z$ and $e^{+} e^{-} \rightarrow h A[11]$.

\section{HIGGS DECAYS INTO SM FERMIONS}

The predictions for the decays $h \rightarrow f \bar{f}$ in the MSSM are affected by two main sources of large corrections which can give rise to large deviations of the MSSM predictions compared to the SM case. The Higgs propagator corrections affect the couplings to fermions in particular via large $h-H$ mixing effects. As a consequence, the effective $h f \bar{f}$ coupling can be heavily suppressed in certain regions of the parameter space $[9,12,13]$. This is shown in the left plot of Fig. 1 , where $\operatorname{BR}(h \rightarrow b \bar{b})$ is given as a function of $M_{\mathrm{A}}$ for $M_{\mathrm{SUSY}}=X_{\mathrm{t}}=500 \mathrm{GeV}$ and $\mu=-1 \mathrm{TeV}\left(M_{\mathrm{SUSY}}\right.$ is the squark mass scale and $X_{\mathrm{t}}$ is the off-diagonal entry in the $\tilde{\mathrm{t}}$ mixing matrix, see Ref. [2]). The plot shows that for a given value of $M_{\mathrm{A}}$ the theoretical prediction for $\operatorname{BR}(h \rightarrow b \bar{b})$ can change drastically if a part of the corrections is neglected. This is illustrated here by neglecting the two-loop contributions or the momentum dependence of the Higgs propagator corrections (the latter corresponds to the $\alpha_{\text {eff }}$ approximation). As a consequence, in these regions of parameter space an accurate theoretical prediction is very difficult to achieve.
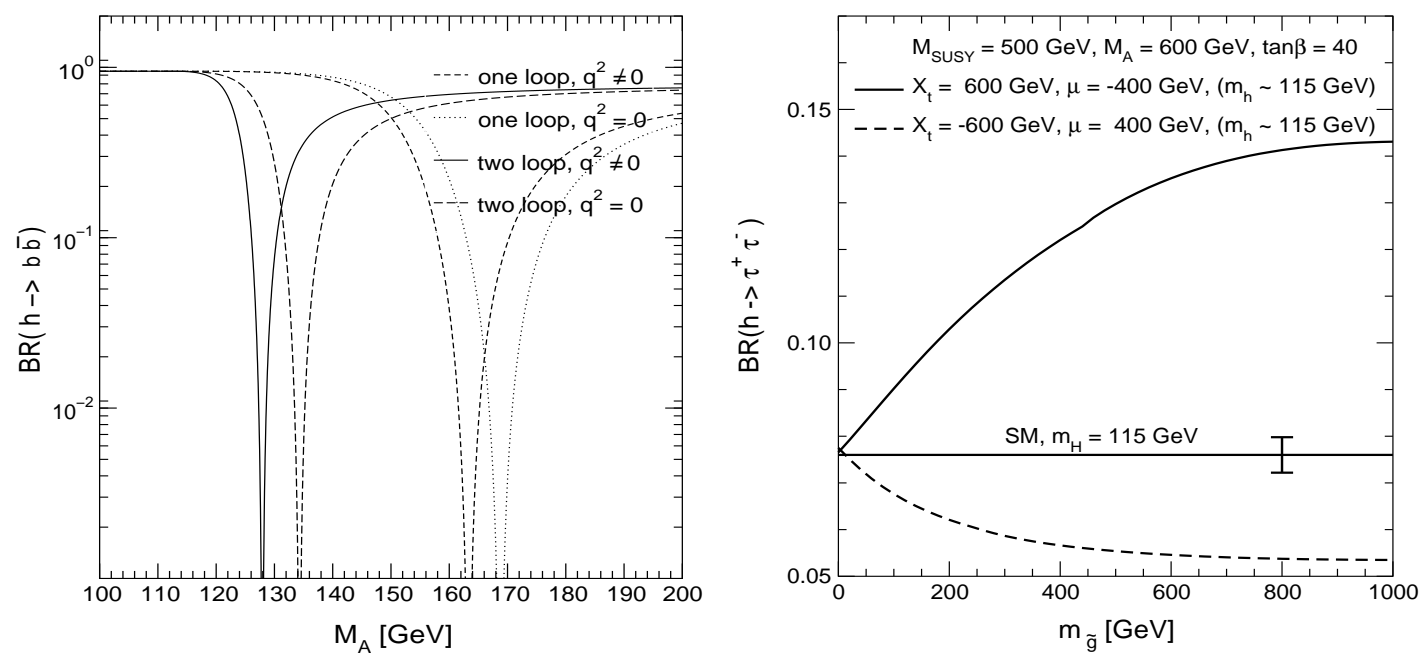

FIGURE 1. Effects of large SUSY contributions on $\mathrm{BR}(h \rightarrow b \bar{b})$ (left plot) and $\operatorname{BR}\left(h \rightarrow \tau^{+} \tau^{-}\right)$ (right plot). The error bar at the SM prediction in the right plot indicates the prospective experimental accuracy at a future LC.

While the Higgs propagator corrections affect the Higgs couplings to all uptype fermions and to all down-type fermions in a universal way, these couplings can be shifted relative to each other by large gluino and higgsino loop corrections. Corrections of this kind can occur for large values of $\tan \beta$ and/or $\mu$. They affect the 
tree-level relation between the fermion masses (in particular $m_{b}$ and $m_{\tau}$ ) and the Yukawa couplings [14]. A deviation in the ratio of the $h b \bar{b}$ and the $h \tau^{+} \tau^{-}$couplings from the SM value caused by large gluino corrections to $\Gamma(h \rightarrow b \bar{b})$ can give rise to a sizable shift in $\operatorname{BR}\left(h \rightarrow \tau^{+} \tau^{-}\right)$. A precise measurement of $\operatorname{BR}\left(h \rightarrow \tau^{+} \tau^{-}\right)$at a future LC will thus provide a high sensitivity for a distinction between the SM and the MSSM even for relatively large values of $M_{\mathrm{A}}$, where otherwise the Higgs sector behaves mainly SM-like. This is illustrated in the right plot of Fig. 1, where $\mathrm{BR}\left(h \rightarrow \tau^{+} \tau^{-}\right)$in the MSSM is shown as a function of the gluino mass, $m_{\tilde{\mathrm{g}}}$, in comparison with the SM prediction and the prospective experimental accuracy at a future LC of about $5 \%$.

\section{HIGGS PRODUCTION IN HIGGS-STRAHLUNG AND ASSOCIATED PRODUCTION}

In Fig. 2 the predictions for the production cross sections $e^{+} e^{-} \rightarrow h Z, h A$ based on combining the complete diagrammatic one-loop result in the MSSM [10] with the dominant two-loop Higgs-propagator corrections (evaluated with the program FeynHiggs [15]) are shown as a function of $m_{\mathrm{h}}$ for $\sqrt{s}=500 \mathrm{GeV}, M_{\mathrm{SUSY}}=1 \mathrm{TeV}$, the slepton mass scale $M_{\tilde{\mathrm{I}}}=300 \mathrm{GeV}, M_{\mathrm{A}}=200 \mathrm{GeV}$ and $X_{\mathrm{t}} / M_{\mathrm{SUSY}}=2$, i.e. maximal mixing in the scalar top sector. The result including the two-loop Higgspropagator corrections is compared with the one-loop result, and the effect of the one-loop box contributions is shown separately. The FD result is furthermore compared with an improved Born approximation, where only corrections to $m_{\mathrm{h}}$ and $\alpha_{\text {eff }}$ are taken into account, which are evaluated within the renormalizationgroup-improved one-loop EPA with the program subhpole (based on Refs. [4,16]).

As can be seen in the figure, the inclusion of the two-loop contributions has a very large effect. The deviation between the full result and the improved Born approximation based on the renormalization-group-improved one-loop EPA is also significant, exceeding $20 \%$ for $\sigma_{\mathrm{Ah}}$. The box contributions change the total cross section by $5-10 \%$ at LC collider energies, and in general give even larger corrections

to the differential cross sections. For the prospective experimental accuracies at a future LC these corrections thus need to be included in the theoretical predictions.

\section{REFERENCES}

1. H. Haber and R. Hempfling, Phys. Rev. Lett. 66 (1991) 1815;

Y. Okada, M. Yamaguchi and T. Yanagida, Prog. Theor. Phys. 85 (1991) 1;

J. Ellis, G. Ridolfi and F. Zwirner, Phys. Lett. B 257 (1991) 83; Phys. Lett. B 262 (1991) 477; R. Barbieri and M. Frigeni, Phys. Lett. B 258 (1991) 395.

2. S. Heinemeyer, W. Hollik and G. Weiglein, Eur. Phys. Jour. C 9 (1999) 343.

3. S. Heinemeyer, W. Hollik and G. Weiglein, Phys. Rev. D 58 (1998) 091701; Phys. Lett. B 440 (1998) 296; Phys. Lett. B 455 (1999) 179. 

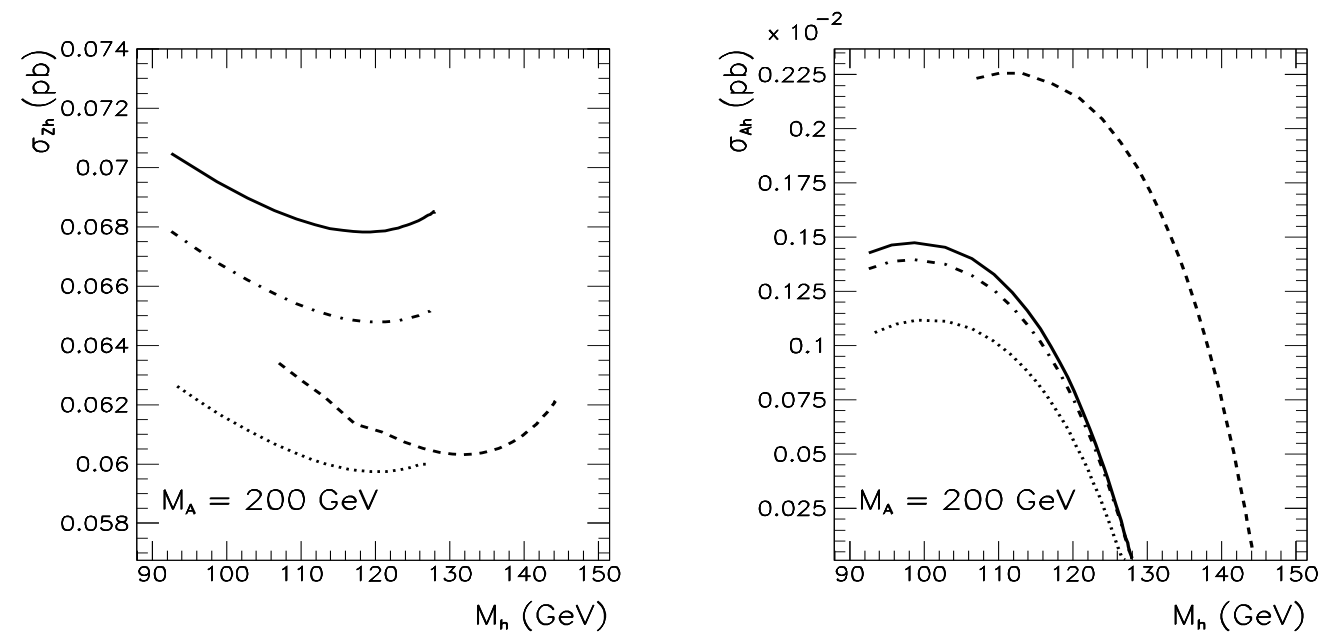

FIGURE 2. $\sigma_{\mathrm{Zh}}$ and $\sigma_{\mathrm{Ah}}$ as a function of $m_{\mathrm{h}}$ at $\sqrt{s}=500 \mathrm{GeV}$ for maximal mixing in the scalar top sector. The solid line represents the FD result consisting of the complete one-loop and the two-loop Higgs-propagator corrections. The dot-dashed line indicates the corresponding result where the box contributions have been omitted, the dashed line shows the one-loop FD result, and the dotted line represents an improved Born approximation evaluated within the renormalization-group-improved one-loop EPA.

4. M. Carena, J. Espinosa, M. Quirós and C. Wagner, Phys. Lett. B 355 (1995) 209;

M. Carena, M. Quirós and C. Wagner, Nucl. Phys. B 461 (1996) 407.

5. H. Haber, R. Hempfling and A. Hoang, Z. Phys. C 75 (1997) 539.

6. J.R. Espinosa and R. Zhang, Nucl. Phys. B 586 (2000) 3.

7. S. Heinemeyer, W. Hollik and G. Weiglein, JHEP 06 (2000) 009.

8. E. Braaten and J.P. Leveille, Phys. Rev. D 22 (1980) 715; N. Sakai, Phys. Rev. D 22 (1980) 2220; T. Inami and T. Kubota, Nucl. Phys. B 179 (1981) 171; A. Dabelstein, W. Hollik, Z. Phys.C 53 (1991) 507; A. Dabelstein, Nucl. Phys. B 456 (1995) 25.

9. S. Heinemeyer, W. Hollik and G. Weiglein, Eur. Phys. Jour. C 16 (2000) 139.

10. P. Chankowski, S. Pokorski and J. Rosiek, Phys. Lett. B 274 (1992) 191; Phys. Lett. B 286 (1992) 307; Nucl. Phys. B 423 (1994) 437; Nucl. Phys. B 423 (1994) 497;

A. Dabelstein, Z. Phys. C 67 (1995) 495;

V. Driesen and W. Hollik, Zeit. Phys. C 68 (1995), 485.

11. S. Heinemeyer, W. Hollik, J. Rosiek and G. Weiglein, hep-ph/0102081.

12. W. Loinaz and J. Wells, Phys. Lett. B 445 (1998) 178.

13. M. Carena, S. Mrenna and C. Wagner, Phys. Rev. D 60 (1999) 075010.

14. H. Eberl, K. Hidaka, S. Kraml, W. Majerotto, Y. Yamada, Phys. Rev. D 62 (2000) 055006; M. Carena, D. Garcia, U. Nierste, C. Wagner, Nucl. Phys. B 577 (2000) 88.

15. S. Heinemeyer, W. Hollik and G. Weiglein, Comput. Phys. Commun. 124 (2000) 76; CERN-TH/2000-055, hep-ph/0002213.

16. M. Carena, H. Haber, S. Heinemeyer, W. Hollik, C. Wagner and G. Weiglein, Nucl. Phys. B 580 (2000) 29. 